\title{
MEETING OF SOUTH GERMAN LARYNGOLOGISTS IN HEIDELBERG.
}

(From the "Münchener Med. Wochenschrift.")

\author{
Dr. Alfred Kirstein (Berlin). Autoscopy of the Air Passages. \\ (See Abstracts.)
}

Dr. Avellis (Frankfurt). Acute Empyema of the Antrum of Highmore, and its Spontaneous Cure.

Having pointed out that acute empyema is much more common than is generally supposed, and having indicated why so many cases are not recognized, the author cited some of his own cases. Zarniko's statement that the symptoms of acute empyema correspond to those of the chronic cases, is not at all correct. Edema of the cheek and eyelids, or of the cheek alone, is an important symptom. From his own experience, and from the cases reported elsewhere, Avellis draws the following conclusions :-

1. Acute empyema of the antrum is very common.

2. There are severe and slight cases. Of the latter several have been reported in England; of severe cases I could find no reports, although, doubtless, many must have been observed.

3. The characteristic symptoms of the slight cases are: painful sensations of pressure and tension in the upper jaw ; purulent, sometimes hæmorrhagic, discharge, of an irregular atypical character; the pain is increased by pressure, by coughing, or by straining; the secretion does not cease entirely during the night; soft œdematous swellings of the cheeks and eyelids frequently occur; the odematous part is at times bright red ; supra-orbital pain is rare, and bad smell is frequently entirely wanting.

4. The severe have all the symptoms of the slight cases, with, in addition, pretty high fever, apathy, photophobia, so great prostration and illness that patient stays entirely in bed, very profuse secretion, nausea, vomiting, mental faculties dull. I have seen such severe cases arise both from influenza, and also spontaneously.

5. Acute empyema readily returns. An ordinary cold in the head is enough to set up a fresh attack.

6. Bilateral empyema was as common (in my experience) as unilateral.

7. I only once saw an acute case pass into the chronic stage.

8. Acute empyema can give rise to polypi.

My opinion with regard to the spontaneous cure of acute antral empyema may be formulated thus :-

Slight cases almost all heal spontaneously; but recurrence is common. This may take place weeks or years later.

(The presence of empyema, and also of the cure, was established in all my cases-myself excepted-by washing out the antrum from the inferior meatus.)

Recovery is gradual. The first sign of improvement is that the pain 
comes on later each day, and gradually diminishes, and the disuharge gets less in quantity and less purulent in character till it finally ceases.

In those cases that require treatment, the antrum is to be syringed out; and it seems to me a matter of indifference whether this is done through the inferior meatus, through the natural ostium, through the alveolus, or through the canine fossa. The rapidity or slowness of cure depends, not on the method of irrigation, but on the nature of the infection. This, again, doubtless depends on the kind of bacteria present; but on this point we as yet know nothing definite.

I hope that my paper may have the effect of putting a stop (1) to foolish and misguiding reports of the rapid cure of empyemata by this or that method of treatment ; $(\because)$ to the mixing together of the symptomatology and prognosis of acute empyema with those of the very widely different chronic condition.

G. Killian (Freiburg). Exploratory Puncture of the Nasal Accessory Cavities.

The only reliable method of diagnosing empyema of an accessory cavity is by syringing or blowing out the cavity. The latter is useful where pus or mucus is present in small quantities, or where the fluid is serous, because in these cases the fluid from the cavity is lost in the fluid from the syringe. The antrum of Highmore can be punctured with a pointed Hartmann's canula in the middle region of the middle meatus. Under cocaine the patient is not aware that anything special has been done, and the danger of wounding the orbit is very small. During three years, during which $I$ and my assistants have made use of this method, we have never wounded the orbit.

The sphenoid sinus can often be sounded, and its natural opening entered by a canula. When this is not the case, its anterior wall can easily be pierced, even with a blunt instrument, as Schäffer has shown. Further, it is not always impossible to enter through the inferior wall with a rightangled instrument.

The ethmoid cells are more difficult to deal with. They all lie along the olfactory slit and can be entered from it, there being only a very thin bony wall to penetrate. In doing this one must keep above an imaginary line drawn from the top of the choana to the anterior end of the root of the middle turbinated. The instrument to use is a canula with its point bent at a right angle, the bent portion being just long enough to penetrate the thin median wall of the cells. With this instrument there is no fear of wounding the orbit.

If the space between septum and middle turbinated is too narrow, one must get at these cells from the middle meatus. When the middle meatus is fairly wide one can reach anterior, middle, or posterior cells with a canula bent upwards at the point at a right angle. If one keeps to the bend of the middle turbinated, avoiding its lateral point of origin, and works straight up, it is impossible to wound the orbit; but to avoid the danger of wounding the cavum cranii the bent portion of the instrument must not be more than half to one centimitre long. By the one route or the other every cell can be reached. 
Only in a small proportion of cases can one sound the frontal sinus without a preliminary operation. Schäffer's method of entering through the floor, from between the middle turbinated and the septum is too dangerous. In most cases the anterior end of the middle turbinated must first be removed-a simple and generally permissible operation-and thus the road cleared for a blunt canula.

KLEMPERER (Strasburg). On the Bacteriology of the Nose.

StClair Thomson and Hewlett have shown that in about eighty cases the cavity of the nose is germ-free, and that only the vestibule contains numerous bacteria. Klemperer does not agree with them, but maintains that in healthy noses, while it is true that bacteria are to be found in quantity only in the vestibule, still no part of the nose is germ-free. Let the anterior parts of the nose be thoroughly sterilized with perchloride of mercury and washed out with sterilized water, then wipe eut the parts higher and deeper in with sterilized cotton-wool swabs. These (the swabs) always bring away a few germs, from which two, three, four, or more frequently six, eight, ten colonies can be cultivated.

Klemperer cannot confirm the statements of Wurtz and Lermoyez as to the bactericidal properties of nasal mucus. Unlike these authors, he experimented not with anthrax bacillus, but with the bacteria which he had previously cultivated from the nose whose mucus he was testing. At first they did not grow well, and even diminished to some extent in number, but soon grew accustomed to the mucus and multiplied in it.

Extinction was never observed.

JURASZ (Heidelberg) presented a patient with a simple Tracheal Polypus.

Hedderich. Clinical Experiences with Paramonochlor-Phenol in Laryngeal Phthisis.

A year ago Dr. Spengler of St. Petersburg warmly recommended this drug. He experimented with it on pure cultures of tubercle bacillus, testing these afterwards on guinea-pigs ; further he applied it to twentysix patients, and reported ten cures.

We have treated thirty patients with paramonochlor-phenol in Professor Jurasz $\mathrm{s}$ clinic, and can report fair results. All the patients admitted that their condition had improved, usually after the first two applications. The dysphagia and irritability of the throat specially disappeared, and breathing became freer ; ulcers became clean, and slowly healed; œdema and infiltration gradually grew less. In serious progressive cases no improvement took place. Two cases seemed to be cured, but as we have not seen them lately we cannot speak of them with any certainty. In three cases the treatment had to be given up, because nausea and vomiting came on regularly after each application of the drug. Along with this treatment general treatment was used, but purposely all operations were avoided.

Paramonochlor-phenol is phenol in which one $H$ is replaced by one Cl. ; it is little soluble in water, but freely in glycerine. We used a ten per cent. solution in glycerine for the larynx, a twenty per cent. for the 
nose and pharynx; the latter causes a white slough, like acid. carbol. liquefact.

While the results are by no means brilliant, they justify further investigation and trial of the drug.

LUBIINski (Berlin) had triel chlor-phenol, but had given it up again; its advantages were outweighed by its disadvantages.

SeIFERT (Würzburg) had given it up as far as the larynx was concerned, but still advised its use in tuberculosis or lupus of the nose.

PROEUSTING (Wiesbaden). On Operations for Malignant Tumours of the Naso-pharynx.

After some introductory remarks the author reported a case of nasopharyngeal fibro-sarcoma in a peasant of nineteen, which he and Herr Sanitätsrath Cramer had had under their care.

The patient had suffered during the winter from repeated, violent epistaxis, and in March consulted Dr. Scheben. He removed several polypi from the nose, but, owing to the violent hæmorrhage this operation set up, had to send the patient to hospital. The author then saw patient, found the left naris blocked, and left side of naso-pharynx greatly narrowed by a tumour. From this he removed with galvanic snare a piece as large as a walnut, which proved to be fibro-sarcomatous. Cramer was called in. He first performed tracheotomy, and introduced a modified Trendelenburg's canula. Then he split the soft palate and the mucous membrane and periosteum of the hard palate, in the middle line, and with a chisel cut off the posterior portion of the hard palate. A free view was thus obtained of the naso-pharynx, and of the nose as far forward as the middle of the inferior turbinated. It then appeared that the tumour could not be removed from below, because it adhered firmly to the upper lateral wall of the nose. Temporary resection of the nose was therefore performed, after firmly packing the naso-pharynx and posterior half of the nose. The skin incision extended from the angle of the right eye, across the root of the nose, down the naso-labial fold, and straight back across the upper lip. Then the nasal bone and the proc. nasal. of the superior maxilla were chiselled through, and the nose turned over to the right. The tumour (about the size of an apple, and having many polypus-like processes) was then seized both from in front and from behind and below, loosened from its base, and shelled out with a large Lorentz's spoon. With this the bleeding, which had been considerable, ceased. The nose was firmly packed with iodoform gauze, and the external wound and that in the soft palate exactly stitched. The tracheal canula was left in till the evening. The result was in every respect satisfactory. As yet no recurrence ; but the operation was done only two months ago.

Discussing the question of recurrence, the author narrated a case observed by Cramer and himself. Cramer had removed a naso-pharyngeal sarcoma from a forty-eight-years-old woman. A few months later this recurred, and grew so rapidly (completely blocking the nose, and both Eustachian tubes, with resulting deafness, and driving forward the left bulbus) that death seemed certain, and was daily expected. Without any apparent reason spontaneous shrinking of the tumour set in, the eye 
returned to its normal position, hearing was restored, and the nose cleared, and the woman is now alive and well (i.e., six years later). The diagnosis of sarcoma had been confirmed by the best microscopists. This tumour must not be confounded with the naso-pharyngeal fibroma of young people, which is probably commoner than is generally supposed.

ROSENFELD (Stuttgart). Demonstration of a Laryngeal Carcinoma.

The patient was a woman eighty-one years old, but very bealthy and strong. Up till March, 1895, she had always been healthy. At that date commenced to suffer from hoarseness and cough, to which a few weeks later was added dysphagia; still later, shortness of breath and stabbing pain in the right ear. I first saw her on 12th September, and diagnosed carcinoma of the larynx, which, originating in the thyroid cartilage, had already spread through the corda vocalis to the arytenoid cartilage. Nothing abnornal to be found external to the larynx. Operation, even tracheotomy, refused. On 14th October tracheotomy permitted and performed. Up till then only fluids had caused pain in swallowing, but thereafter nothing but fluids could be swallowed; by January 14th even they could no longer be taken. Still, life was prolonged (on water and nutrient enemata) till February 15th.

DREYFuss (Strasburg) demonstrated a specimen of Flat-Celled Epithelioma Laryngis, which, apparently originating in the right sinus pyriformis, had perforated the lateral laryngeal wall, and appeared as a granulating tumour above the right false cord. Partial resection. Death four days later from pne'monia. Several cancerous glands as large as cherry stones, which had not been noticed at the operation, were found post-mortem deep in the neck.

KIRSTEIN (Berlin) showed an instrument for Removing the Pharyngeal Tonsil.

KILLIAN (Freiburg) showed a Rheostat for Galziano-Cautery, worked by foot, and enabling the operator to turn the current off or on, and to increase or diminish it, while actually using the cautery or cauterysnare.

It is made by Ellis in Freiburg. Arthur J. Hutchison (Trans. and Abs.).

BERLIN LARYNGOLOGICAL SOCIETY. Meeting, January I7th, 1896. (Reported by Dr. MEYER.)

(Continued from page 225.)

GLUCK presented:-(1) Patient, aged thirty-six, whose larynx had been partially removed for carcinoma. (2) Patient, aged fifty-six, whose larynx had been entirely removed. Gluck recommends the procedure he adopted in this case-viz., to stitch the trachea to the skin after it has been divided transversely ; in this way the aspiration of secretion from 